\title{
Leishmania amazonensis Infection: A Comparison of in Vivo Leishmanicidal Mechanisms between Immunized and Naive Infected $B A L B / c$ Mice
}

\author{
M. L. Pompeu, ${ }^{1}$ L. A. Freitas, ${ }^{*}$ M. L. V. Santos, ${ }^{*}$ A. Barral, and \\ M. BARRAL-NETto ${ }^{2}$
}

Faculdade de Medicina da Universidade Federal da Bahia R. Joao das Botas, sin, and *Centro de Pesquisas Goncalo Moniz, 40.140 Salvador-Bahia, Brazil

\begin{abstract}
Pompeu, M. L., Freitas, L. A., Santos, M. L. V., Barral, A., and Barral-Netto, M. 1992. Leishmania amazonensis infection: A comparison of in vivo leishmanicidal mechanisms between immunized and naive infected BALB/c mice. Experimental Parasitology 74, 169-176. In vitro studies have shown that both macrophage activation and destruction of parasitized macrophages lead to leishmania destruction. The relative role played by such mechanisms in vivo have not been properly evaluated. We took advantage of the model of intravenous immunization with solubilized leishmanial antigen which renders partially resistant the otherwise highly susceptible $B A L B / c$ mice to address this issue avoiding the interference of different genetic backgrounds. Leishmania destruction occurred in three situations: destruction of the parasitized macrophage, which were in close contact with lymphocytes or eosinophils; extracellular damage, always surrounded by small foci of granulocytes; and parasite damage inside activated macrophages. Destruction of the parasitized macrophages was frequently seen in immunized and protected animals. Our observations suggest that destruction of parasite-loaded macrophages is an important mechanism of host protection in experimental cutaneous leishmaniasis. 1992 Academic Press, Inc.

INDEX DESCRIPTORS AND ABBREVIATIONS: Leishmania amazonensis; Leishmaniasis; Immunization; Leishmania; Tegumentary leishmaniasis; Experimental leishmaniasis.
\end{abstract}

\section{INTRODUCTION}

Resistance in experimental cutancous leishmaniasis has been correlated to an influx of lymphocytes to the lesion, whereas very few lymphocytes are observed in susceptible animals (McElrath et al. 1987). Activation of macrophages by $\mathrm{T}$ lymphocytes or its products is thought to be the most important host defense mechanism in leishmaniasis. Several in vitro models have shown the destruction of different leishmanial strains inside macrophages stimulated with lymphokines, such as interferon- $\gamma$ (Nathan et al. 1983), or directly activated by contact with lymphocytes (Panosian et

\footnotetext{
1 Present address: Departmento de Patologia, Universidade Federal do Ceara.

${ }^{2}$ To whom correspondence should be addressed at Seattle Biomedical Research Institute, 4 Nickerson St., Seattle, WA 98109.
}

al. 1984). Recently the lysis in vitro of the parasitized cell by lymphocytes has also been described (Pham and Mauel 1987). The relative importance of such mechanisms in vivo has not been evaluated.

Although local reactions are heterogeneous and dynamic, histopathological changes such as necrosis, granuloma formation, and fibrosis have been correlated with protection in humans (Ridley and Ridley 1983) or experimental leishmaniasis comparing resistant and susceptible mouse strains (Andrade et al. 1984). It is difficult, however, to discriminate factors involved in resistance from those merely reflecting diverse genetic backgrounds but not implicated in specific protection. Such difficulty may be overcome by using the same mouse strain altering its susceptibility to leishmania. Extremely susceptible BALB/c animals may be rendered partially resistant to 
leishmanial infection by intravenous immunization (Howard et al. 1982). Using such immunization models for protection against Leishmania amazonensis we have observed different histological pictures after 7 weeks of infection between immunized mice and their susceptible counterparts (Barral-Netto et al. 1987). In this report we investigate in detail at the ultrastructural level the differences between these animals regarding the mechanisms of parasite destruction.

\section{MATERIAL AND Methods}

Animals. Ten- to fourteen-week-old BALB/c mice were obtained from our own colony.

Parasites. Leishmania amazonensis (MHOM/BR/ 76/ Josefa) was used for both infection and antigen preparation. This strain was typed by serodeme and zymodeme analysis, and its behavior in mice has been previously described (Andrade et al. 1984). For immunization stationary-phase promastigotes were solubilized with $0.5 \% \mathrm{NP}-40$ and $0.25 \%$ sodium deoxycholate, in the presence of protease inhibitors as reported elsewhere (Barral-Netto et al. 1987). Concentration of the antigen is expressed as parasite equivalents per milliliter.

Immunization and infection. Mice were immunized intravenously with 3 doses of $5 \times 10^{7}$ parasite equivalents at weekly intervals. One week after the last immunizing dose mice were challenged subcutaneously in the right hind foot-pad with $5 \times 10^{6}$ viable stationary-phase promastigotes. Foot-pad thickness was measured periodically with a dial gauge caliper (C. Starret, Athol, MA), and the difference between the infected and contralateral foot-pad was expressed as lesion size (in milliliters).

Tissue processing. Small fragments from the lesion of four animals per group were obtained at 4,7 , or 10 weeks following infection. The tissue was immediately fixed and processed for electron microscopy as described (Barral-Netto et al. 1987), and other fragments were processed for optical histology being stained in Hematoxilin-Eosin.

Pathologic examination. Criteria used for macrophage activation were presence of microvili and pinocytotic vesicles, expansion of the cytoplasm with a larger number of organelles, decrease of the heterochromatin ring, presence of evident nucleolus, and an increase in number and size of the lysosomes ( $\mathrm{Pa}$ padimitriou and Spector 1971; Black and Epstein 1974; Crawford and Hardwicke 1978; Turk et al. 1978; Van der Rhee et al. 1979; Adams 1976; Adams and Hamilton 1984). Indicators of parasite damage or lysis were those described in the literature as increase in the number and size of multivesicular bodies and lipid goticles, marked dilatation of endoplasmic reticulum, mitochondria, or flagellar pocket, decrease in ribosome number, and rupture of the plasma membrane (Wery and De Groodt-Lasseel, 1966; Alexander and Vickerman, 1975; Hentzer and Kobayase, 1977; Sandhank and Ben-David, 1979).

Quantitative analysis of inflammatory cells. Evaluation of the frequency of cells in the lesion, and their interaction with the parasite, was performed using ultrathin sections with a differential count of 100 to 200 inflammatory cells for each animal. The area(s) where the cells were counted was representative of the histological picture for each animal as indicated by examination of the semi-thin sections.

\section{RESULTS}

Immunized mice were able to control the progression of $L$. amazonensis infection and developed much smaller lesions than unimmunized animals (Fig. 1). Lesions of unimmunized animals exhibited a continuous increase in the percentage of infected macrophages (as a percentage of total cells in the inflammatory infiltrate) reaching $40.3 \%$ at 10 weeks postinfection, whereas immunized animals had between 14.7 and $17.1 \%$ from 3 to 10 weeks postinfection (Fig. 1).

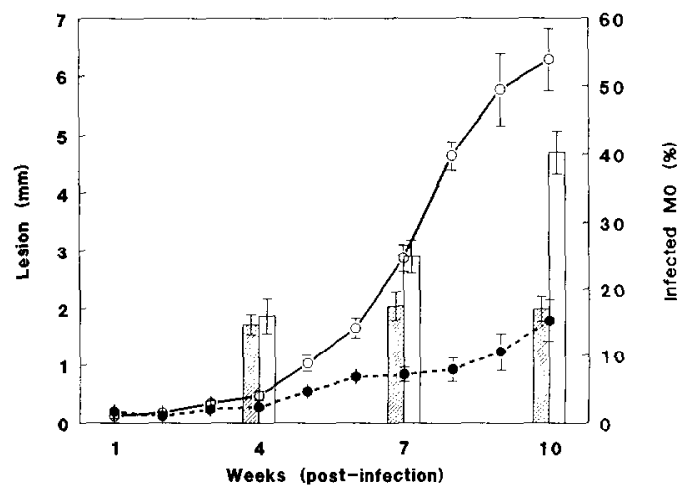

FIG. 1. Kinetics of lesion growth in BALB/c animals infected with $L$. amazonensis without previous immunization (open circles) or after intravenous immunization with solubilized promastigote antigen (closed circles), and infected macrophages as a percentage of total cells in the lesion inflammatory infiltrate in naive (open bars) or immunized (hatched bars) animals. 
At up to 4 weeks of infection lesions of immunized animals exhibited a histological pattern undistinguishable from their unimmunized counterparts; at this time lesion size was also very similar in both groups. The only difference observed was in the frequency of activated macrophages. Activated macrophages (Figs. $2 \mathrm{a}$ and $2 \mathrm{e}$ ) were seen more frequently in the immunized group, whereas nonactivated macrophages (Fig. 2b) were predominantly observed in unimmunized animals. Signs of macrophage activation were observed in $32.5 \%$ of macrophages in the immunized group, whereas only $17.1 \%$ of macrophages exhibited such aspects in unimmunized animals at 4 weeks of infection (Fig. 3, upper panel).

After the seventh week postinfection, when differences in lesion size were apparent between the two groups of animals, the histological pattern reflected such differences. Unimmunized infected controls exhibited the classical monomorphic reaction, composed almost exclusively of vacuolated and heavily parasitized macrophages. The histological picture observed in the immunized group, at this period of infection, comprised a mixed cell inflammatory reaction with scarcely parasitized macrophages and lymphocytes. At the 10th week postinfection a similar, only more marked, pattern was observed in both groups.

Immunized animals exhibited a continuous increase in the percentage of activated macrophages (Fig. 3, upper panel) and in the presence of cell destruction (Fig. 3, lower panel), whereas in lesions from unimmunized animals these findings remained constant (Fig. 3, upper and lower panels).

Both well-preserved (Fig. 2c) and damaged (Fig. 2d) parasites were observed inside macrophages. Parasite damage was observed in three main situations. The most frequent damage was the destruction of the parasitized macrophage (Figs. 2f and 4a). Such cells were in close contact with lymphocytes (Fig. 4b), eosinophils (Figure 4c), and occasionally other mononuclear cells with abundant rough endoplasmic reticulum, resembling epithelioid cells. Sometimes the signs of damage were more pronounced in the host cells than in the parasite. It was also observed the presence of amastigotes at the extracellular space, probably released from ruptured macrophages. All parasites seen in the extracellular space were damaged and were surrounded by small foci of granulocytes, with the presence of eosinophils (Figs. 4d and 4e). Extracellular parasites were damaged even when no cell was observed in their vicinity.

The third situation where parasite destruction was observed occurred in the interior of the activated macrophage (Fig. 4f). Such observations were more prominent in the immunized group, which exhibited almost $90 \%$ of its macrophages with signs of intense activation after the seventh week of infection, than in unimmunized animals. Despite the widespread, and intense, macrophage activation observed in the lesions of immunized animals many parasites remained in their interior without signs of damage, and intramacrophage parasite destruction was less frequently seen than the lysis of the parasitized cell in protected animals.

\section{Discussion}

The observations made in the present study clearly demonstrated that histological aspects reflect, and indicate, the course of leishmanial infection in genetically homogeneous mice. The observation of the initial signs of macrophage activation slightly preceded any measurable macroscopical difference between immunized or unimmunized BALB/c infected with Leishmania amazonensis. The increased differences in lesion size between the two groups of animals were paralleled by markedly divergent histological patterns.

Since histological aspects reflect so well the course of infection in these animals it seems probable that the frequency of ob- 

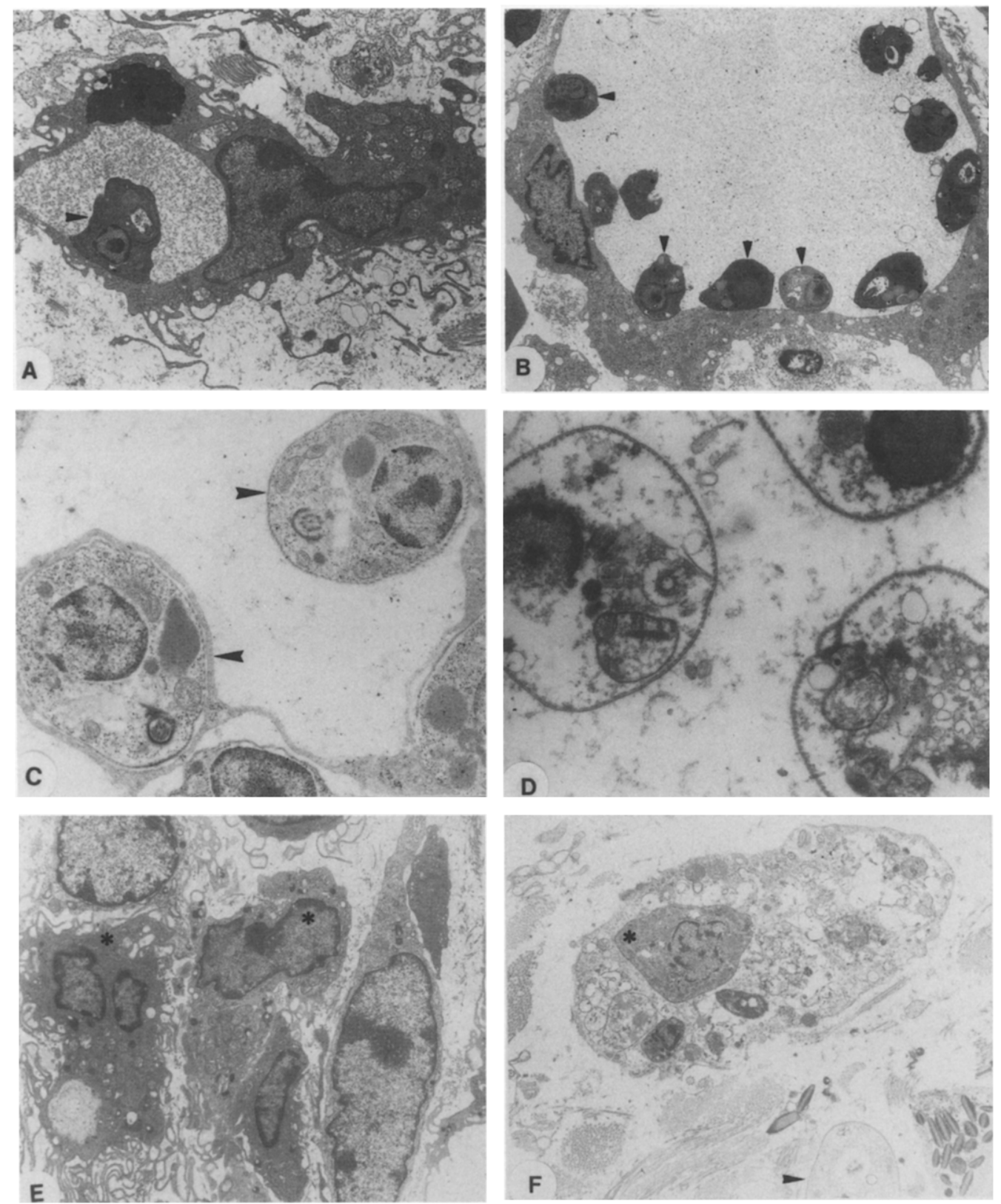

Fig. 2. (a) Activated macrophage from immunized mouse. The cell presents a small parasitophorous vacuole containing one amastigote (arrowhead). Note the ruffled cytoplasmic expansions, indented nucleus, and lysosomes. $\times 3322$. (b) Nonactivated macrophage from a naive infected mouse. Most of the cytoplasm is occupied by a very large parasitophorous vacuole containing undamaged parasites (arrowhead). $\times 2372$. (c) Well-preserved amastigotes (arrows) within a large parasitophorous vacuole. Parasite structures are well preserved. $\times 5513$. (d) Damaged parasites showing cytoplasmic vacuolization, dilatation of organelles, and pyknotic nucleus. $\times 9073$. (e) Well-preserved activated Inacrophages $\left({ }^{*}\right) \times 2372$. (f) Lysed macrophage containing Leishmania $\left({ }^{*}\right)$. Note cytoplasmic organelles dilatation and mitochondrial swelling. In the left bottom there are part of a eosinophil, eosinophil granules in connective tissue, and damaged amastigote (arrowhead) free in the interstitiom. $\times 3322$. 

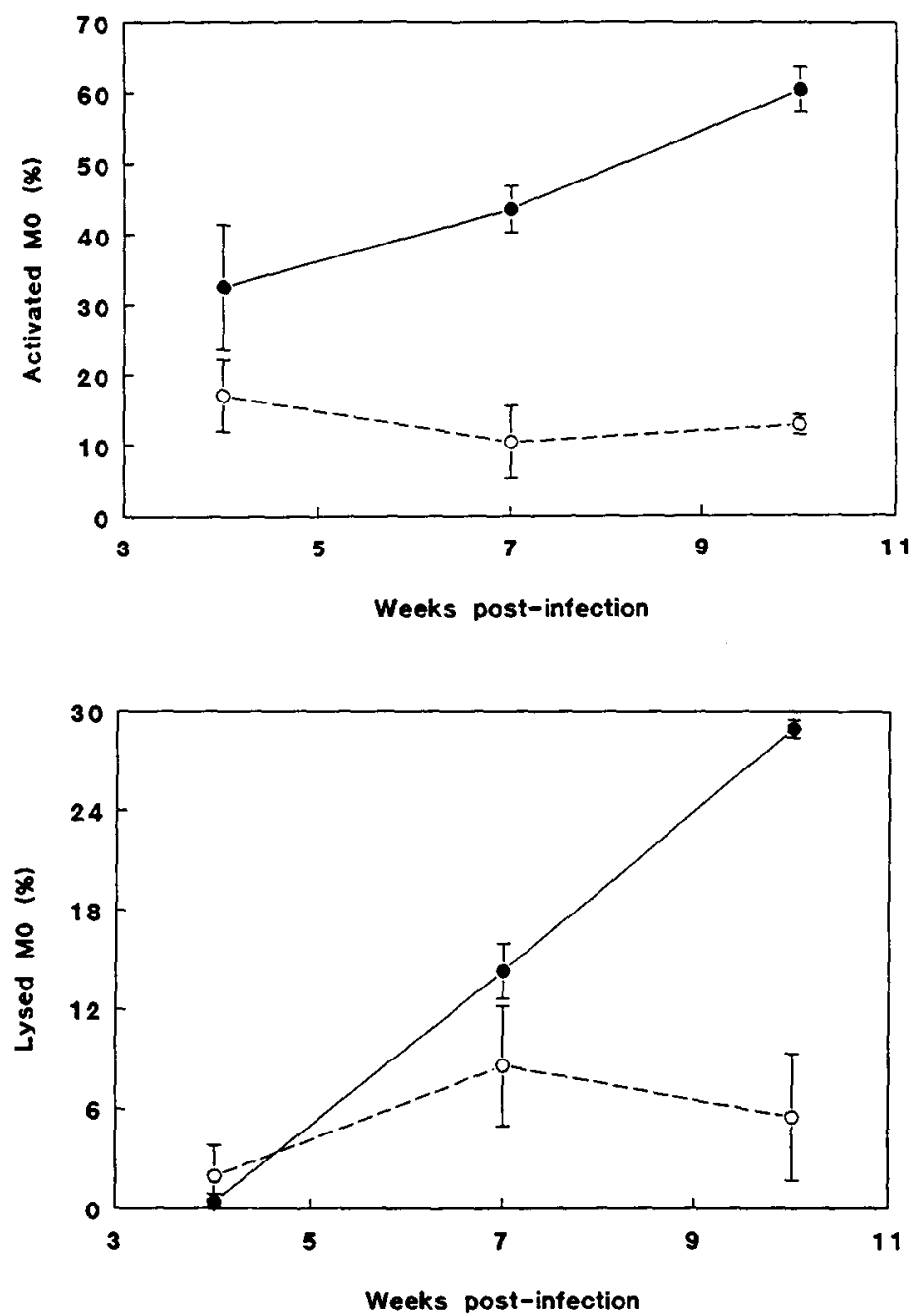

FIG. 3. Upper panel. Percentage of activated macrophages in the lesion inflammatory infiltrate of BALB/c mice infected with $L$. amazonensis without previous immunization (open circles) or after immunization (closed circles). Lower panel. Percentage of lysed macrophages in the same animals as upper panel.

served parasiticidal aspects also reflects the importance of the operative in vivo mechanisms. According to this line of reasoning the destruction of leishmania in the protected animals occurs largely by the destruction of the parasitized macrophage. Macrophage activation, and intracellular parasite destruction, seems also to be an important in vivo protective mechanism, as suggested by in vitro studies.

The importance of $\mathrm{CD}^{+} \mathrm{T}$ cells has been demonstrated in $L$. major-infected mice (Titus et al. 1987; Hill et al. 1989) and particularly in immunized $\mathrm{BALB} / \mathrm{c}$ mice, in which anti-Lyt-2 treatment abrogated the induction of resistance (Farrell et al. 1989). Such findings correlate with in vitro observations of the lysis of leishmania-infected macrophages by specifically sensitized lymphocytes (Pham and Mauel 1987). These findings had already made clear the possibility of parasitized cell destruction 

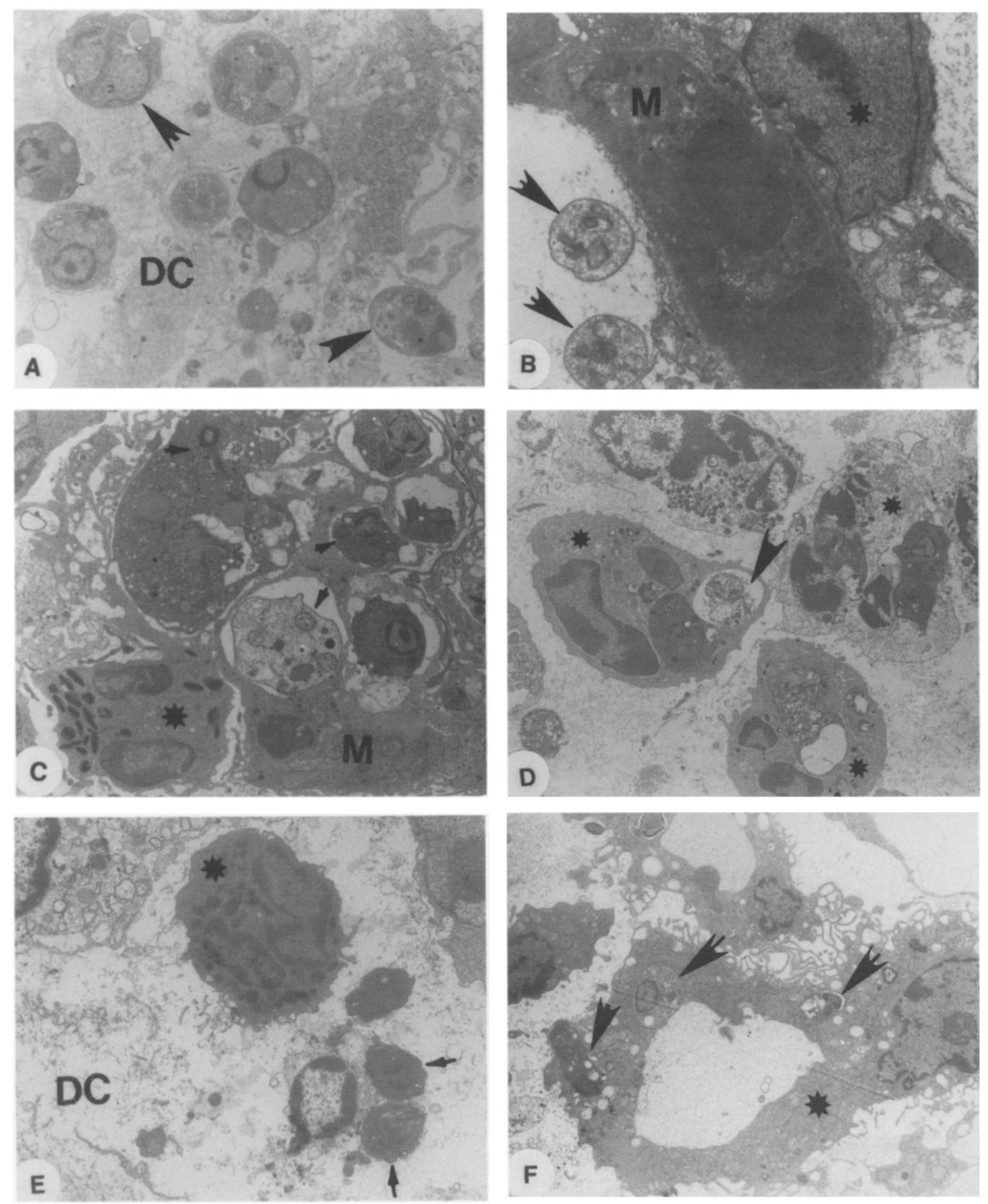

FIG. 4. (a) Area of lytic necrosis of cell. Leishmania are free in the tissue among remains of dead cell (DC). Some amastigotes show degenerative changes $\times 3322$. (b) Degenerated leishmania (arrowheads) within the parasitophorous vacuole of a macrophage $(\mathrm{M})$, which is in close contact with a lymphocyte $\left(^{*}\right) \times 3322$. (c) Close contact between eosinophil $(*)$ and macrophage $(\mathrm{M})$ which contains damaged amastigotes (arrowheads) within parasitophorous vacuole $\times 3322$. (d) Neutrophils (*) phagocytizing leishmania organisms exhibiting variable degree of degenerative changes (arrowheads) $\times 2372$. (e) Remains of dead cell (DC) in a focus of cytolysis. Amastigotes in the extracellular space are damaged (arrows). Note the presence of eosinophil $\left(^{*}\right)$ in their vicinity $\times 2372$. (f) Parasites with variable degrees of degeneration (arrowheads) within activated macrophages $\left({ }^{*}\right) . \times 2372$. 
being a way of controlling intracellular microbial infections, but there were few indications of its occurrence in real in vivo situations (Hancock et al. 1989), and how relevant this mechanism was as compared to others, such as macrophage activation. Our data give support to the idea of parasite elimination via macrophage destruction being an important element of host protection in murine cutaneous leishmaniasis. Both macrophage activation and lysis of the infected cells occur during leishmaniasis, and based on histopathological evidences, they are important mechanisms of parasite elimination, leading to protection against leishmanial infection in mice.

\section{ACKNOWLEDGMENTS}

Supported by grants from the Brazilian National Research Council (CNPq) and US-PHS (Grant AI 16282).

\section{REFERENCES}

ADAMS, D. O. 1976. The granulomatous inflammatory response. A review. American Journal of Pathology 84, 164 .

Adams, D. O., and Hamilton, T. A. 1984. The biology of macrophage activation. Annual Review of Immunology 2, 283-318.

Alexander, J., AND Vickerman, K. 1975. Fusion of the host cell secondary lysosomes with the parasitophorous vacuoles of Leishmania mexicana-infected macrophages. Journal of Protozoology 22, 502.

Andrade, Z. A., ReEd, S. G., Roters, S. B., AND SADIGURSKY, M. 1984. Immunopathology of experimental cutaneous leishmaniasis. American Journal of Pathology 144, 137.

Barral-Netto, M., Freitas, I. A. R., and ANDRADE, Z. A. 1987. Histopathological changes induced by vaccination in experimental cutaneous leishmaniasis of BALB/c mice. American Journal of Pathology 127, 271-278.

Barral-Netto, M., ReEd, S. G., Sadigursky, M., AND SONNENFELd, G. 1987. Specific immunization with solubilized antigen in experimental American cutaneous leishmaniasis. Clinical and Experimental Immunology 67, 11-19.

Black, M. M., AND EpsteIn, W. L. 1974. Formation of multinucleated giant cells in organized epithelioid cell granulomas. American Journal of Pathology 74, 263.

Crawford, C. L., and Hardwicke, P. M. D. 1978. Ultrastructural features of epithelioid cell granu- loma induced by intradermal injection of xenogeneic nerve tissue. Journal of Pathology 125, 107.

Farrell, J. P., Muller, I., ANd Louis, J. A. 1989. A role for $\mathrm{Lyt}-2^{+} \mathrm{T}$ cells in resistance to cutaneous leishmaniasis in immunized mice. Journal of Immunology 142, 2052-2956.

Hancock, G. E., Cohn, Z. A., and Kaplan, G. 1989. The generation of antigen-specific, major histocompatibility complex-restricted cytotoxic $\mathrm{T}$ lymphocytes of the $\mathrm{CD} 4^{+}$phenotype. Enhancement by the cutaneous administration of interleukin-2. Journal of Experimental Medicine 169, 909-919.

Hentzer, B., and Kobayase, T. 1977. The ultrastructure of Leishmania tropica in skin lesions. Acta Pathologica et Microbiologica Scandinavica Section $B 85,153$.

Hill, J. O., Awwad, M., ANd North, R. J. 1989. Elimination of CD4 suppressor $T$ cells from susceptible BALB/c mice releases CD8 $\mathrm{T}$ lymphocytes to mediate protective immunity against Leishmania. Journal of Experimental Medicine 169, 1819-1827.

Howard, J. G., Nicklin, S., Hale, C., and Liew, F. Y. 1982. Prophylactic immunization against experimental leishmaniasis. 1. Protection induced in mice genetically vulnerable to fatal Leishmania tropica infection. Journal of Immunology 129, 1329 1333.

McElrath, M. J., Kaplan, G., Nusrat, A., and CoHN, Z. A. 1987. Cutaneous leishmaniasis. The defect in $\mathrm{T}$ cell influx in BALB/c mice. Journal of Experimental Medicine 165, 546-559.

Nathan, C. F., Murray, H. W., Wiebe, M. E., AND RUBIN, B. Y. 1983. Identification of interferon- $\gamma$ as the lymphokine that activates human macrophages oxidative metabolism and antimicrobial activity. Journal of Experimental Medicine 158, 670-689.

Panosian, C. B., Sypek, J. P., ANd Wyler, D. J, 1984. Cell contact-mediated macrophage activation for anti-leishmanial defense. I. Lymphocyte effector mechanism that is contact dependent and noncytotoxic. Journal of Immunology 133, 3358-3365.

Papadimitriou, J. M., and Spector, W. G. 1971. The origin properties and fate of epithelioid cells. Journal of Pathology 105, 187.

Pham, T. V., AND Mauel, J. 1987. Studies on intracellular killing of Leishmania major and lysis of host macrophages by immune lymphoid cells in vitro. Parasite Immunology 9, 721-736.

Ridley, D. S., ANd Ridley, M. J. 1983. The evolution of the lesion in cutaneous leishmaniasis. International Journal of Dermatology 18, 50.

SandBank, M., AND Bf.N-Davin, E. 1979. The role of lisosomes in the healing process of cutaneous leishmaniasis. International Journal of Dermatology 18, 50. 
Titus, R. G., Milon, G., Marchal, G., Vassalli, P., Cerottini, J. C., and Louis, J. A. 1987. Involvement of specific Lyt-2 ${ }^{+} \mathrm{T}$ cells in the immunological control of experimentally induced murine cutaneous leishmaniasis. European Journal of Immunology 17, 1429-1433.

Turk, J. L., Jones, B., AND PARker, D. 1978. Ultrastructural observations of epithelioid cell granulomas induced by zirconium in the guinea pig. Journal of Pathology 125, 45.

Van Der Rhee, H. J., Van De-Burgh, W., and
Deams, W. TH. 1979. The differentiation of monocytes into macrophages epithelioid cells, and multinucleated giant cells in the subcutaneous granulomas. Cell and Tissue Research 197, 355.

Wery, M., AND De GroodT-Lasseel, M. 1966. Ultrastructure de Trypanosoma cruzi en culture sur milieu semi-synthetique. Annales de la Sciete Belge de Medicine Tropicale 46, 337.

Received 21 June 1991; accepted with revision 29 October 1991 